\title{
BINOMIAL EDGE IDEALS OF GENERALIZED BLOCK GRAPHS
}

\author{
ARVIND KUMAR
}

\begin{abstract}
We classify generalized block graphs whose binomial edge ideals admit a unique extremal Betti number. We prove that the Castelnuovo-Mumford regularity of binomial edge ideals of generalized block graphs is bounded below by $m(G)+1$, where $m(G)$ is the number of minimal cut sets of the graph $G$ and obtain an improved upper bound for the regularity in terms of the number of maximal cliques and pendant vertices of $G$.
\end{abstract}

\section{INTRODUCTION}

Let $R=K\left[x_{1}, \ldots, x_{m}\right]$ be a standard graded polynomial ring over an arbitrary field $K$, and $M$ be a finitely generated graded $R$-module. Let

$$
0 \longrightarrow \bigoplus_{j \in \mathbb{Z}} R(-j)^{\beta_{p, j}^{R}(M)} \stackrel{\phi_{p}}{\longrightarrow} \cdots \stackrel{\phi_{1}}{\longrightarrow} \bigoplus_{j \in \mathbb{Z}} R(-j)^{\beta_{0, j}^{R}(M)} \stackrel{\phi_{0}}{\longrightarrow} M \longrightarrow 0
$$

be the minimal graded free resolution of $M$, where $p \leq m$ and $R(-j)$ is the free $R$-module of rank 1 generated in degree $j$. The number $\beta_{i, j}^{R}(M)$ is called the $(i, j)$-th graded Betti number of $M$. The projective dimension and Castelnuovo-Mumford regularity (henceforth called regularity) are two invariants associated with $M$ that can be read off from the minimal graded free resolution of $M$. The regularity of $M$, denoted by $\operatorname{reg}(M)$, is defined as

$$
\operatorname{reg}(M):=\max \left\{j-i \mid \beta_{i, j}^{R}(M) \neq 0\right\},
$$

and the projective dimension of $M$, denoted by $\operatorname{pd}_{R}(M)$, is defined as

$$
\operatorname{pd}_{R}(M):=\max \left\{i: \beta_{i, j}^{R}(M) \neq 0\right\} .
$$

A Betti number $\beta_{i, j}^{R}(M) \neq 0$ is called an extremal Betti number if $\beta_{r, s}^{R}(M)=0$ for all pairs $(r, s) \neq(i, j)$ with $r \geq i$, and $s \geq j$. Observe that $M$ admits a unique extremal Betti number if and only if $\beta_{p, p+r}^{R}(M) \neq 0$, where $p=\operatorname{pd}_{R}(M)$ and $r=\operatorname{reg}(M)$. For a graded $R$-module $M$, we denote the Betti polynomial of $M$ by

$$
B_{M}(s, t)=\sum_{i, j} \beta_{i, j}^{R}(M) s^{i} t^{j} .
$$

Herzog et al. in [9] and independently Ohtani in [20] introduced the notion of binomial edge ideal corresponding to a finite simple graph. Let $G$ be a simple graph on $[n]$. Let $S=K\left[x_{1}, \ldots, x_{n}, y_{1}, \ldots, y_{n}\right]$, where $K$ is a field. The binomial edge ideal of $G$ is $J_{G}=$ $\left(x_{i} y_{j}-x_{j} y_{i}:\{i, j\} \in E(G), i<j\right)$. Researchers have found exact formulas or bounds for algebraic invariants of $J_{G}$, such as codimension, depth, Betti numbers and regularity, in terms of combinatorial invariants of the underlying graph $G$, see e.g., [1, 2, 3, 5, 15, 17, 19, 25, 26]. The study of regularity and Betti numbers of homogeneous ideals has attracted a lot of

Key words and phrases. Binomial edge ideal; Castelnuovo-Mumford regularity; Generalized block graph; Extremal Betti number.

Mathematics Subject Classification 2010: 13D02, 13C13, 05E40. 
attention in the recent past due to its algebraic and geometric importance. In [19], Matsuda and Murai proved that if $G$ is a graph on the vertex set $[n]$, then $\ell(G) \leq \operatorname{reg}\left(S / J_{G}\right) \leq n-1$, where $\ell(G)$ is the length of a longest induced path in $G$. In particular, they conjectured, and later proved by Kiani and Saaedi Madani [15], that $\operatorname{reg}\left(S / J_{G}\right)=n-1$ if and only if $G$ is a path on $n$ vertices. Another conjectured upper bound for $\operatorname{reg}\left(S / J_{G}\right)$ is given by $\operatorname{cl}(G)$, the number of maximal cliques of $G$ [26]. The latter conjecture has been recently proved for chordal graphs by Rouzbahani Malayeri et al. [24], extending results of [6, 12, 25], and for some classes of non-chordal graphs in [16].

An interesting class of chordal graphs consists of block graphs, which are connected graphs whose blocks (i.e., maximal subgraphs that cannot be disconnected by removing a vertex) are cliques. Recently, in [11], Herzog and Rinaldo improved the lower bound for the regularity of binomial edge ideals of block graphs and classified block graphs whose binomial edge ideal admits a unique extremal Betti number. In this article, we extend these results to the class of generalized block graphs that contains block graphs. We also obtain improved lower and upper bounds for the regularity of binomial edge ideals of these graphs.

The article is organized as follows. In the second section, we recall some results on graphs and binomial edge ideals. In the third section, we characterize generalized block graphs whose binomial edge ideal admits a unique extremal Betti number (Theorem [3.11). In particular, we prove that $\beta_{p(G), p(G)+m(G)+1}^{S}\left(S / J_{G}\right)$ is an extremal Betti number of $S / J_{G}$ if $G$ is a connected generalized block graph (Theorem [3.7), where $p(G)=\operatorname{pd}_{S}\left(S / J_{G}\right)$ and $m(G)$ is the number of minimal cut sets of $G$. As a consequence, we obtain $\operatorname{reg}\left(S / J_{G}\right) \geq m(G)+1$ (Corollary 3.12). In the fourth section, we obtain improved upper bound for the regularity of binomial edge ideals of generalized block graphs in terms of the number of maximal cliques and pendant vertices of $G$ (Theorem 4.5).

\section{PRELiminaries}

In this section, we recall some notation and terminology from graph theory, and some important results about binomial edge ideals.

Let $G$ be a finite simple graph with vertex set $V(G)$ and edge set $E(G)$. For $A \subseteq V(G)$, $G[A]$ denotes the induced subgraph of $G$ on the vertex set $A$, that is the subgraph with edge set $E(G[A])=\{\{i, j\} \in E(G): i, j \in A\}$. For a vertex $v, G \backslash v$ denotes the induced subgraph of $G$ on the vertex set $V(G) \backslash\{v\}$. A vertex $v \in V(G)$ is said to be a cut vertex if $G \backslash v$ has more connected components than $G$. For $T \subset[n]$, let $\bar{T}=[n] \backslash T, c_{G}(T)$ be the number of connected components of $G[\bar{T}]$ and $c_{G}$ the number of connected components of $G$. We say that a subset $T \subset[n]$ is a cut set of $G$ if $c_{G}(T)>c_{G}$. A cut set of $G$ is said to be a minimal cut set if it is minimal under inclusion. A subset $U$ of $V(G)$ is said to be a clique if $G[U]$ is a complete graph.

Let $\Delta$ be a simplicial complex on the vertex set $[n]$ and $\mathcal{F}(\Delta)$ be the set of its facets. A facet $F$ of $\Delta$ is called a leaf if either $F$ is the only facet of $\Delta$ or else there exists a facet $G$, called a branch of $F$, such that for each facet $H$ of $\Delta$ with $H \neq F, H \cap F \subseteq G \cap F$.

The simplicial complex $\Delta$ is called a quasi-forest if its facets can be ordered as $F_{1}, \ldots, F_{s}$ such that for all $i>1$, the facet $F_{i}$ is a leaf of the simplicial complex with facets $F_{1}, \ldots, F_{i-1}$. Such an order of the facets is called a leaf order. The simplicial complex whose facets are the maximal cliques of a graph $G$ is called the clique complex of $G$ and denoted by $\Delta(G)$. By [8, Theorem 9.2.12], $G$ is chordal if and only if $\Delta(G)$ is a quasi-forest. 
A vertex $v$ of $G$ is said to be a free vertex if $v$ belongs to exactly one maximal clique of $G$. A vertex $v$ is said to be an internal vertex of $G$ if it is not a free vertex. The set $N_{G}(v)=\{u \in V(G):\{u, v\} \in E(G)\}$ is called the neighborhood of $v$, and $G_{v}$ denotes the graph on the vertex set $V(G)$ and edge set $E\left(G_{v}\right)=E(G) \cup\left\{\{u, w\}: u, w \in N_{G}(v)\right\}$. Observe that, if $v$ is a free vertex, then $G_{v}=G$.

Let $G_{1}, \ldots, G_{c_{G}(T)}$ be the connected components of $G[\bar{T}]$. For each $i$, let $\widetilde{G}_{i}$ denote the complete graph on $V\left(G_{i}\right)$ and $P_{T}(G)=\left(\bigcup_{i \in T}\left\{x_{i}, y_{i}\right\}, J_{\widetilde{G_{1}}}, \ldots, J_{\widetilde{G_{c_{G}}(T)}}\right)$. In [9], it was shown by Herzog et al. that $J_{G}=\bigcap_{T \subseteq[n]} P_{T}(G)$. For each $i \in T$, if $i$ is a cut vertex of the graph $G[\bar{T} \cup\{i\}]$, then we say that $T$ has the cut point property. Set $\mathcal{C}(G)=\{\emptyset\} \cup\{T$ : $T$ has the cut point property\}. It follows from [9, Corollary 3.9] that $T \in \mathcal{C}(G)$ if and only if $P_{T}(G)$ is a minimal prime of $J_{G}$. Hence, by [9, Theorem 3.2] and [9, Corollary 3.9], we have $J_{G}=\bigcap_{T \in \mathcal{C}(G)} P_{T}(G)$.

\section{Extremal Betti number of Generalized Block Graphs}

In this section, we study the extremal Betti number $\beta_{p(G), p(G)+j}^{S}\left(S / J_{G}\right)$ of binomial edge ideals of generalized block graphs, where $p(G)=\operatorname{pd}_{S}\left(S / J_{G}\right)$. A maximal connected subgraph of $G$ with no cut vertex is called a block. A graph $G$ is called a block graph if each block of $G$ is a clique. In other words, a block graph is a chordal graph such that every pair of blocks of $G$ intersects in at most one vertex. Block graphs were extensively studied by many authors, see [5], [6], [11], [13].

Generalized block graphs are the generalization of block graphs and were introduced in [14]. A chordal graph $G$ is said to be a generalized block graph if $F_{i}, F_{j}, F_{k} \in \mathcal{F}(\Delta(G))$ such that $F_{i} \cap F_{j} \cap F_{k} \neq \emptyset$, then $F_{i} \cap F_{j}=F_{i} \cap F_{k}=F_{j} \cap F_{k}$. One could see that all block graphs are generalized block graphs. By definition of generalized block graph, it is clear that a subset $A$ of vertices of $G$ is a minimal cut set if and only if there exist $F_{t_{1}}, \ldots, F_{t_{q}} \in \mathcal{F}(\Delta(G))$ such that $\bigcap_{j=1}^{q} F_{t_{j}}=A$, and for all other facets $F$ of $\Delta(G), F \cap A=\emptyset$. Note that if $A$ is a minimal cut set, then $A$ is a clique. For a minimal cut set $A$, we denote by $G_{A}$ the graph obtained from $G$ by replacing the cliques $F_{t_{1}}, \ldots, F_{t_{q}}$ with the clique on the vertex set $\underset{j \in[q]}{\cup} F_{t_{j}}$.

Lemma 3.1. Let $G$ be a graph on the vertex set $[n]$ and $A$ be a minimal cut set of $G$. Then $A \in \mathcal{C}(G)$. Moreover, if $G$ is a generalized block graph, then for every $T \in \mathcal{C}(G)$, either $A \subseteq T$ or $A \cap T=\emptyset$.

Proof. For the first claim, suppose that $A \notin \mathcal{C}(G)$. Then there exists $v \in A$ such that $c_{G}(A \backslash\{v\})=c_{G}(A)$. Since $A$ is a minimal cut set, we know that $c_{G}(A)>c_{G}$, and hence, $c_{G}(A \backslash\{v\})>c_{G}$. This means that $A \backslash\{v\}$ is a cut set and is properly contained in $A$, against the minimality of $A$. Thus, $A \in \mathcal{C}(G)$. The second claim clearly holds when $|A|=1$. Let $|A| \geq 2$ and $T \in \mathcal{C}(G)$. If $T \cap A=\emptyset$, then there is nothing to prove. Assume that $T \cap A \neq \emptyset$ and let $v \in T \cap A$. Then $v$ is a cut vertex of $G[\bar{T} \cup\{v\}]$. Suppose that there exists $w \in A \backslash T$. We want to show that $N_{G}(v)=N_{G}(w)$. Suppose that there exists $u \in N_{G}(w) \backslash N_{G}(v)$ and let $F$ be a facet of $\Delta(G)$ containing $w$ and $u$. Since $A$ is a cut set, there are at least two facets $F_{1}, F_{2}$ containing $v, w$ but not $u$. Then, we have $F_{1} \cap F_{2} \cap F \neq \emptyset, v \in F_{1} \cap F_{2}$ but $v \notin F_{1} \cap F$ and $v \notin F_{2} \cap F$, against the fact that $\mathrm{G}$ is a generalized block graph. Thus, $N_{G}(w) \subset N_{G}(v)$. Similarily, $N_{G}(v) \subset N_{G}(w)$, and hence, $N_{G}(v)=N_{G}(w)$. Consequently, $v$ is not a cut vertex of $G[\bar{T} \cup\{v\}]$, which is a contradiction. Hence, $A \subseteq T$. 
We now give an example of a chordal graph $G$ that is not a generalized block graph for which the second claim of Lemma 3.1, does not hold.

Example 3.2. Let $G$ be a graph as shown in Fig. 1. Then, it can be seen that $G$ is a chordal graph that is not a generalized block graph. The sets $A=\{2,3\}$ and $B=\{3,4\}$ are minimal cut sets of $G$, thus $A, B \in \mathcal{C}(G)$, and $A \cap B \neq \emptyset$. However, $A \nsubseteq B$ and $B \nsubseteq A$.

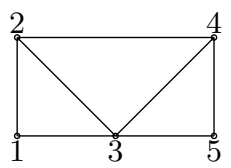

\section{FiguRE 1}

Let $G$ be a generalized graph on $[n]$. Let $A$ be a minimal cut set of $G$. Set

$$
Q_{1}=\bigcap_{\substack{T \subseteq[n] \\ A \cap T=\emptyset}} P_{T}(G) \quad, \quad Q_{2}=\bigcap_{\substack{T \subseteq[n] \\ A \subseteq T}} P_{T}(G) .
$$

By [9, Theorem 3.2, Corollary 3.9], $J_{G_{A}}=\bigcap_{T \subset[n]} P_{T}\left(G_{A}\right)=\bigcap_{T \in \mathcal{C}\left(G_{A}\right)} P_{T}\left(G_{A}\right)$. It follows from [22, Proposition 2.1] that $T \in \mathcal{C}\left(G_{A}\right)$ if and only if $A \cap T=\emptyset$ and $T \in \mathcal{C}\left(G_{A}[\bar{A}]\right)$. If $A \cap T=\emptyset$, then $P_{T}(G)=P_{T}\left(G_{A}\right)$. Consequently, $Q_{1}=J_{G_{A}}$. Note that

$$
Q_{2}=\left(x_{i}, y_{i}: i \in A\right)+\bigcap_{T \backslash A \subset[n] \backslash A} P_{T \backslash A}(G[\bar{A}])=\left(x_{i}, y_{i}: i \in A\right)+J_{G[\bar{A}]},
$$

where the last equality follows from [9, Theorem 3.2]. Thus,

$$
Q_{1}+Q_{2}=\left(x_{i}, y_{i}: i \in A\right)+J_{G_{A}[\bar{A}]} .
$$

By virtue of Lemma 3.1, $J_{G}=Q_{1} \cap Q_{2}$. This gives us the following short exact sequence,

$$
0 \longrightarrow \frac{S}{J_{G}} \longrightarrow \frac{S}{Q_{1}} \oplus \frac{S}{Q_{2}} \longrightarrow \frac{S}{Q_{1}+Q_{2}} \longrightarrow 0 .
$$

The following example illustrates that, in general, $Q_{1} \neq J_{G_{A}}$ for a minimal set $A$ of $G$.

Example 3.3. Let $G$ be a graph as shown in Fig. 11. Then, $T \in \mathcal{C}(G)$ if and only if $T \in\{\emptyset,\{2,3\},\{3,4\}\}$. Set $A=\{2,3\}$ and $B=\{3,4\}$. Note that $A$ is a minimal cut set of $G$ and $G_{A}=G \cup\{1,4\}$. Let $T \subseteq[5]$ such that $A \cap T=\emptyset$. Then, $T \subseteq\{1,4,5\}$, and hence, $P_{\emptyset}(G) \subseteq P_{T}(G)$. Thus,

$$
Q_{1}=\bigcap_{T \subseteq\{1,4,5\}} P_{T}(G)=P_{\emptyset}(G)=J_{K_{5}} \neq J_{G_{A}} .
$$

However,

$$
\begin{aligned}
Q_{2} & =\bigcap_{T \subseteq[5], A \subset T} P_{T}(G)=\left(x_{2}, y_{2}, x_{3}, y_{3}\right)+\bigcap_{T \subseteq\{1,4,5\}} P_{T}(G[\{1,4,5\}]) \\
& =\left(x_{2}, y_{2}, x_{3}, y_{3}, x_{4} y_{5}-x_{5} y_{4}\right)=P_{A}(G) .
\end{aligned}
$$

Since $J_{G}=P_{\emptyset}(G) \cap P_{A}(G) \cap P_{B}(G)$, we have $J_{G} \neq Q_{1} \cap Q_{2}$. 
Let $p(G)$ denote the projective dimension of $S / J_{G}$. Then $\operatorname{pd}_{S}\left(S / Q_{1}\right)=p\left(G_{A}\right)$. Let $T=K\left[x_{1}, \ldots, x_{n}\right]$. Let $0<m<n, I \subset R=K\left[x_{1}, \ldots, x_{m}\right]$ and $J \subset R^{\prime}=K\left[x_{m+1}, \ldots, x_{n}\right]$ be homogeneous ideals. Then, the minimal graded free resolution of $T /(I+J)$ is the tensor product of the minimal free resolutions of $R / I$ and $R^{\prime} / J$. For $A \subset[n]$, set $S_{A}=K\left[x_{i}, y_{i}: i \notin\right.$ $A]$. Hence, $\operatorname{pd}_{S}\left(S / Q_{2}\right)=2|A|+\operatorname{pd}_{S_{A}}\left(S_{A} / J_{G[\bar{A}]}\right)=2|A|+p(G[\bar{A}])$ and $\operatorname{pd}_{S}\left(S /\left(Q_{1}+Q_{2}\right)\right)=$ $2|A|+\operatorname{pd}\left(S_{A} / J_{G_{A}[\bar{A}]}\right)=2|A|+p\left(G_{A}[\bar{A}]\right)$.

In [14], Kiani and Saeedi Madani obtained the depth of binomial edge ideals of generalized block graphs, and hence, their projective dimension by the Auslander-Buchsbaum formula. Recall that the clique number of a graph $\mathrm{G}$, denoted $\omega(G)$, is the maximum size of the maximal cliques of $G$. Let $G$ be a generalized block graph on $[n]$. For each $i=1, \ldots, \omega(G)-1$, we set

$$
\mathcal{A}_{i}(G)=\{A \subseteq[n]:|A|=i, A \text { is a minimal cut set of } G\}
$$

and $a_{i}(G)=\left|\mathcal{A}_{i}(G)\right|$. Observe that a generalized block graph $G$ is a block graph if and only if $a_{i}(G)=0$, for all $i>1$. Let $m(G)$ denote the number of minimal cut sets of $G$. Then, $m(G)=\sum_{i=1}^{\omega(G)-1} a_{i}(G)$.

By [14, Theorem 3.2] and the Auslander-Buchsbaum formula, it follows that:

Theorem 3.4. Let $G$ be a generalized block graph on $[n]$. Then,

$$
p(G)=n-c_{G}+\sum_{i=2}^{\omega(G)-1}(i-1) a_{i}(G),
$$

where $c_{G}$ is the number of connected components of $G$.

We recall the notion of decomposability from [22, Section 2] and [23]. A graph $G$ is called decomposable if there exist subgraphs $G_{1}$ and $G_{2}$ such that $G=G_{1} \cup G_{2}, V\left(G_{1}\right) \cap V\left(G_{2}\right)=\{v\}$ and $v$ is a free vertex of both $G_{1}$ and $G_{2}$.

A graph $G$ is called indecomposable if it is not decomposable. Up to ordering, $G$ has a unique decomposition into indecomposable subgraphs, i.e., there exist $G_{1}, \ldots, G_{r}$ indecomposable induced subgraphs of $G$ with $G=G_{1} \cup \cdots \cup G_{r}$ such that for each $i \neq j$, either $V\left(G_{i}\right) \cap V\left(G_{j}\right)=\emptyset$ or $V\left(G_{i}\right) \cap V\left(G_{j}\right)=\{v\}$ and $v$ is a free vertex of both $G_{i}$ and $G_{j}$.

In [11, Proposition 3], Herzog and Rinaldo proved that:

Proposition 3.5. [11, Proposition 1.3] Let $G=G_{1} \cup G_{2}$ be a decomposable graph. Let $S_{i}=K\left[x_{j}, y_{j}: j \in V\left(G_{i}\right)\right]$, for $i=1,2$. Then,

$$
B_{S / J_{G}}(s, t)=B_{S_{1} / J_{G_{1}}}(s, t) B_{S_{2} / J_{G_{2}}}(s, t) .
$$

It follows from Proposition 3.5 that if $G=G_{1} \cup \cdots \cup G_{r}$ is a decomposition of $G$ into indecomposable graphs, then $\operatorname{reg}\left(S / J_{G}\right)=\sum_{i \in[r]} \operatorname{reg}\left(S_{i} / J_{G_{i}}\right)$ and $p(G)=\sum_{i \in[r]} p\left(G_{i}\right)$. Also, if for each $i, \beta_{p\left(G_{i}\right), p\left(G_{i}\right)+j_{i}}^{S_{i}}\left(S_{i} / J_{G_{i}}\right)$ is an extremal Betti number of $S_{i} / J_{G_{i}}$, then

$$
\beta_{p(G), p(G)+j}^{S}\left(S / J_{G}\right)=\prod_{i \in[r]} \beta_{p\left(G_{i}\right), p\left(G_{i}\right)+j_{i}}^{S_{i}}\left(S_{i} / J_{G_{i}}\right)
$$

is an extremal Betti number of $S / J_{G}$, where $j=j_{1}+\cdots+j_{r}$. Therefore, it is enough to find the position of the extremal Betti number $\beta_{p(G), p(G)+i}^{S}\left(S / J_{G}\right)$ for indecomposable graphs. 
Lemma 3.6. Let $G$ be a connected indecomposable generalized block graph and let $F_{1}, \ldots, F_{r}$ be a leaf order of $\mathcal{F}(\Delta(G))$. Denote by $F_{t_{1}}, \ldots, F_{t_{q}}$ all the branches of the leaf $F_{r}$. Set $A=F_{r} \cap \bigcap_{i=1}^{q} F_{t_{i}}$ and $\alpha=|A|$. Then,

(a) the graphs $G_{A}, G_{A}[\bar{A}]$ and $G[\bar{A}]$ are generalized block graphs.

(b) for $i \neq \alpha, a_{i}\left(G_{A}\right)=a_{i}(G)$ and $a_{\alpha}\left(G_{A}\right)=a_{\alpha}(G)-1$. In particular,

$$
m\left(G_{A}\right)=m(G)-1 \text { and } p\left(G_{A}\right)=p(G)-\alpha+1 .
$$

(c) for $i \neq \alpha, a_{i}\left(G_{A}[\bar{A}]\right)=a_{i}(G)$ and $a_{\alpha}\left(G_{A}[\bar{A}]\right)=a_{\alpha}(G)-1$. In particular,

$$
m\left(G_{A}[\bar{A}]\right)=m(G)-1 \text { and } p\left(G_{A}[\bar{A}]\right)=p(G)-2 \alpha+1 .
$$

(d) for $i \neq \alpha, a_{i}(G[\bar{A}]) \leq a_{i}(G)$ and $a_{\alpha}(G[\bar{A}]) \leq a_{\alpha}(G)-1$. In particular,

$$
m(G[\bar{A}]) \leq m(G)-1 \text { and } p(G[\bar{A}])=p(G)-2 \alpha-q+1 .
$$

Proof. (a) This easily follows by the fact that $G$ is a generalized block graph.

(b) Notice that for $i \neq \alpha, \mathcal{A}_{i}\left(G_{A}\right)=\mathcal{A}_{i}(G)$ and $\mathcal{A}_{\alpha}\left(G_{A}\right)=\mathcal{A}_{\alpha}(G) \backslash\{A\}$. Thus, by Proposition 3.4, $p\left(G_{A}\right)=n-1+\sum_{i=2}^{\omega\left(G_{A}\right)-1}(i-1) a_{i}\left(G_{A}\right)=p(G)-\alpha+1$ and $m\left(G_{A}\right)=m(G)-1$.

(c) Notice that for $i \neq \alpha, \mathcal{A}_{i}\left(G_{A}[\bar{A}]\right)=\mathcal{A}_{i}(G)$ and $\mathcal{A}_{\alpha}\left(G_{A}[\bar{A}]\right)=\mathcal{A}_{\alpha}(G) \backslash\{A\}$. Thus, by Proposition 3.4, $p\left(G_{A}[\bar{A}]\right)=(n-\alpha)-1+\sum_{i=2}^{\omega\left(G_{A}[\bar{A}]\right)-1}(i-1) a_{i}\left(G_{A}[\bar{A}]\right)=p(G)-2 \alpha+1$ and $m\left(G_{A}[\bar{A}]\right)=\sum_{i=1}^{\omega\left(G_{A}[\bar{A}]\right)-1} a_{i}\left(G_{A}[\bar{A}]\right)=m(G)-1$.

(d) Let $B$ be a minimal cut set of $G[\bar{A}]$. Since $G[\bar{A}]$ is an induced subgraph of $G$ and $B \cap A=\emptyset, B$ is a minimal cut set of $G$. Therefore, for $i \neq \alpha, \mathcal{A}_{i}(G[\bar{A}]) \subseteq \mathcal{A}_{i}(G)$ and $\mathcal{A}_{\alpha}(G[\bar{A}]) \subseteq \mathcal{A}_{\alpha}(G) \backslash\{A\}$. Thus, $m(G[\bar{A}])=\sum_{i=1}^{\omega(G[\bar{A}])-1} a_{i}(G[\bar{A}]) \leq m(G)-1$ and by Proposition 3.4. $p(G[\bar{A}])=(n-\alpha)-(q+1)+\sum_{i=2}^{\omega(G[\bar{A}])-1}(i-1) a_{i}(G[\bar{A}]) \leq p(G)-2 \alpha-q+1$.

Recall that a vertex $v$ is said to be an internal vertex of $G$ if it is not a free vertex. For $v \in V(G)$, let $\operatorname{cdeg}_{G}(v)$ denote the number of maximal cliques of $G$ which contains $v$. The number of free vertices of $G$ is denoted by $f(G)$.

Theorem 3.7. Let $G$ be a connected indecomposable generalized block graph on the vertex set $[n]$. Then, $\beta_{p(G), p(G)+m(G)+1}^{S}\left(S / J_{G}\right)$ is an extremal Betti number of $S / J_{G}$. Moreover, if $G$ is a complete graph or for every internal vertex $v, \operatorname{cdeg}_{G}(v)>2$, then $\beta_{p(G), p(G)+m(G)+1}^{S}\left(S / J_{G}\right)=$ $f(G)-1$.

Proof. We prove this assertion by induction on $m(G)$. If $m(G)=0$, then $G$ is a complete graph. Therefore, the claim follows by the Eagon-Northcott resolution [4]. Assume that $m(G)>0$. Since $G$ is a chordal graph, by [8, Theorem 9.2.12], $\Delta(G)$ is a quasi-forest. Let $F_{1}, \ldots, F_{r}$ be a leaf order of $\mathcal{F}(\Delta(G))$. Let $F_{t_{1}}, \ldots, F_{t_{q}}$ be all the branches of the leaf $F_{r}$. Note that $q \geq 1$. Since $G$ is a generalized block graph, $F_{r} \cap F_{t_{i}}=F_{t_{j}} \cap F_{t_{k}}$ for every pair of $i, j, k \in[q]$ with $j \neq k$ and for all $l \neq t_{1}, \ldots, t_{q}, F_{r} \cap F_{l}=\emptyset$. Let $A=F_{r} \cap F_{t_{1}}=\cap_{i=1}^{q} F_{t_{i}} \cap F_{r}$ and $\alpha=|A|$. Since $A$ is a minimal cut set, by the discussion after Lemma 3.1, $J_{G}=Q_{1} \cap Q_{2}$, where $Q_{1}=J_{G_{A}}$ and $Q_{2}=\left(x_{i}, y_{i}: i \in A\right)+J_{G[\bar{A}]}$.

By Lemma 3.6, $G_{A}, G_{A}[\bar{A}]$ and $G[\bar{A}]$ are generalized block graphs. We have the following cases:

Case (1): If $\alpha=1$, then it follows from Theorem 3.4 and Lemma 3.6 that $p\left(G_{A}\right)=$ $p(G), p\left(G_{A}[\bar{A}]\right)=p(G)-1$ and $p(G[\bar{A}]) \leq p(G)-q-1($ where $G[\bar{A}]$ has $q+1$ connected components). Note that $G[\bar{A}]$ is not necessarily indecomposable, but we can split it into 
smaller indecomposable graphs. Since $G$ is an indecomposable graph, $q \geq 2$, and hence, $\operatorname{pd}_{S}\left(S / Q_{2}\right)=2+p(G[\bar{A}]) \leq p(G)-1$. Therefore,

$$
\operatorname{Tor}_{i}^{S}\left(\frac{S}{Q_{2}}, K\right)=0, \text { for } i \geq p(G) .
$$

Thus, for each $j \geq 0$, the exact sequence (11) yields the long exact sequence of Tor sequence:

$$
\begin{aligned}
0 \rightarrow \operatorname{Tor}_{p(G)+1, p(G)+j}^{S}\left(\frac{S}{Q_{1}+Q_{2}}, K\right) & \rightarrow \operatorname{Tor}_{p(G), p(G)+j}^{S}\left(\frac{S}{J_{G}}, K\right) \rightarrow \\
& \rightarrow \operatorname{Tor}_{p(G), p(G)+j}^{S}\left(\frac{S}{J_{G_{A}}}, K\right) \rightarrow \ldots
\end{aligned}
$$

Since $Q_{1}+Q_{2}=\left(x_{i}, y_{i}: i \in A\right)+J_{G_{A}[\bar{A}]}$, we have that

$$
\operatorname{Tor}_{p(G)+1, p(G)+j}^{S}\left(\frac{S}{Q_{1}+Q_{2}}, K\right) \cong \operatorname{Tor}_{p\left(G_{A}[\bar{A}]\right), p\left(G_{A}[\bar{A}]\right)+(j-1)}^{S_{A}}\left(\frac{S_{A}}{J_{G_{A}[\bar{A}]}}, K\right)
$$

where $S_{A}=K\left[x_{i}, y_{i}: i \notin A\right]$. It follows from induction that

$$
\operatorname{Tor}_{p\left(G_{A}[\bar{A}]\right), p\left(G_{A}[\bar{A}]\right)+(j-1)}^{S_{A}}\left(\frac{S}{J_{G_{A}[\bar{A}]}}, K\right)=0 \quad \text { for } \quad j>m\left(G_{A}[\bar{A}]\right)+2=m(G)+1
$$

and

$$
\operatorname{Tor}_{p(G), p(G)+j}^{S}\left(\frac{S}{J_{G_{A}}}, K\right)=0 \quad \text { for } \quad j>m\left(G_{A}\right)+1=m(G) .
$$

Now, (2), (3) and (4) imply that

$$
\operatorname{Tor}_{p(G), p(G)+j}^{S}\left(\frac{S}{J_{G}}, K\right)=0 \quad \text { for } \quad j>m(G)+1,
$$

and

$$
\operatorname{Tor}_{p\left(G_{A}[\bar{A}]\right), p\left(G_{A}[\bar{A}]\right)+m\left(G_{A}[\bar{A}]\right)+1}^{S_{A}}\left(\frac{S_{A}}{J_{G_{A}[\bar{A}]}}, K\right) \cong \operatorname{Tor}_{p(G), p(G)+m(G)+1}^{S}\left(\frac{S}{J_{G}}, K\right)
$$

By induction, $\beta_{p\left(G_{A}[\bar{A}]\right), p\left(G_{A}[\bar{A}]\right)+m\left(G_{A}[\bar{A}]\right)+1}^{S_{A}}\left(S_{A} / J_{G_{A}[\bar{A}]}\right) \neq 0$ is an extremal Betti number. Now, Eq. ([6) implies

$$
\beta_{p(G), p(G)+m(G)+1}^{S}\left(S / J_{G}\right) \neq 0,
$$

and by Eq. (5), we get that $\beta_{p(G), p(G)+m(G)+1}^{S}\left(S / J_{G}\right)$ is an extremal Betti number.

Case (2): If $\alpha \geq 2$, then by virtue of Theorem 3.4 and Lemma 3.6, $p\left(G_{A}\right)=p(G)-\alpha+1$, $p\left(G_{A}[\bar{A}]\right)=p(G)-2 \alpha+1$. Therefore,

$$
\operatorname{Tor}_{i}^{S}\left(\frac{S}{Q_{1}}, K\right)=\operatorname{Tor}_{i}^{S}\left(\frac{S}{J_{G_{A}}}, K\right)=0, \text { for } i \geq p(G) \text {. }
$$

Note that $G[\bar{A}]$ has $q+1$ connected components. By Theorem 3.4 and Lemma 3.6, we have that $p(G[\bar{A}]) \leq p(G)-2 \alpha-q+1$. Therefore, $\operatorname{pd}_{S}\left(S / Q_{2}\right)=2 \alpha+p(G[\bar{A}]) \leq p(G)-q+1$. 
Thus, for each $j \geq 0$, the exact sequence (11) yields the long exact sequence of Tor sequence:

$$
\begin{aligned}
0 \rightarrow \operatorname{Tor}_{p(G)+1, p(G)+j}^{S}\left(\frac{S}{Q_{1}+Q_{2}}, K\right) & \rightarrow \operatorname{Tor}_{p(G), p(G)+j}^{S}\left(\frac{S}{J_{G}}, K\right) \rightarrow \\
& \rightarrow \operatorname{Tor}_{p(G), p(G)+j}^{S}\left(\frac{S}{Q_{2}}, K\right) \rightarrow \ldots
\end{aligned}
$$

We now distinguish between two sub-cases.

Case (2.1): If $\operatorname{pd}_{S}\left(S / Q_{2}\right)=2 \alpha+p(G[\bar{A}]) \leq p(G)-1$, then

$$
\operatorname{Tor}_{p(G)}^{S}\left(\frac{S}{Q_{2}}, K\right)=0
$$

For each $j \geq 0$, (7) yields that

$$
\operatorname{Tor}_{p(G)+1, p(G)+j}^{S}\left(\frac{S}{Q_{1}+Q_{2}}, K\right) \cong \operatorname{Tor}_{p(G), p(G)+j}^{S}\left(\frac{S}{J_{G}}, K\right) .
$$

Now, Eqs. (3), (44) and (8) imply

$$
\operatorname{Tor}_{p(G), p(G)+j}^{S}\left(\frac{S}{J_{G}}, K\right)=0 \quad \text { for } \quad j>m(G)+1,
$$

and

$$
\operatorname{Tor}_{p\left(G_{A}[\bar{A}]\right), p\left(G_{A}[\bar{A}]\right)+m\left(G_{A}[\bar{A}]\right)+1}^{S_{A}}\left(\frac{S_{A}}{J_{G_{A}[\bar{A}]}}, K\right) \cong \operatorname{Tor}_{p(G), p(G)+m(G)+1}^{S}\left(\frac{S}{J_{G}}, K\right)
$$

By induction, $\beta_{p\left(G_{A}[\bar{A}]\right), p\left(G_{A}[\bar{A}]\right)+m\left(G_{A}[\bar{A}]\right)+1}\left(S_{A} / J_{G_{A}[\bar{A}]}\right) \neq 0$ is an extremal Betti number. Hence, Eq. (10) implies

$$
\beta_{p(G), p(G)+m(G)+1}^{S}\left(S / J_{G}\right) \neq 0
$$

and by Eq. (9), we get that $\beta_{p(G), p(G)+m(G)+1}^{S}\left(S / J_{G}\right)$ is an extremal Betti number.

Case (2.2): If $\operatorname{pd}_{S}\left(S / Q_{2}\right)=p(G)$, then $q=1$. Let $H_{1}$ and $H_{2}$ be connected components of $G[\bar{A}]$. Then, $m(G[\bar{A}])=m\left(H_{1}\right)+m\left(H_{2}\right)$. For $i=1$, 2 , set $S_{H_{i}}=K\left[x_{j}, y_{j}: j \in V\left(H_{i}\right)\right]$. If $H_{2}$ is an isolated vertex, then $\operatorname{pd}_{S}\left(S / Q_{2}\right)=2 \alpha+p\left(H_{1}\right)=2 \alpha+p(G[\bar{A}])$ and $m\left(H_{1}\right)=m(G[\bar{A}]) \leq$ $m(G)-1$. If $H_{2}$ is a non-trivial graph, then $\operatorname{pd}_{S}\left(S / Q_{2}\right)=2 \alpha+p\left(H_{1}\right)+p\left(H_{2}\right)=2 \alpha+p(G[\bar{A}])$ and $m\left(H_{1}\right)+m\left(H_{2}\right)+2=m(G[\bar{A}])+2 \leq m(G)+1$. By induction,

$$
\operatorname{Tor}_{p(G[\bar{A}]), p(G[\bar{A}])+j}^{S_{A}}\left(\frac{S}{J_{G[\bar{A}]}}, K\right)=0 \quad \text { for } \quad j>m(G)+1 \geq m(G[\bar{A}])+2 .
$$

Thus, $\operatorname{Tor}_{p(G), p(G)+j}^{S}\left(\frac{S}{Q_{2}}, K\right)=0$, for $j>m(G)+1$. Now, Eqs. (3), (44) and (7) imply

$$
\operatorname{Tor}_{p(G), p(G)+j}^{S}\left(\frac{S}{J_{G}}, K\right)=0 \quad \text { for } \quad j>m(G)+1 .
$$

By induction, $\beta_{p\left(G_{A}[\bar{A}]\right), p\left(G_{A}[\bar{A}]\right)+m\left(G_{A}[\bar{A}]\right)+1}\left(S_{A} / J_{G_{A}[\bar{A}]}\right) \neq 0$. Consequently, by Eq. (17),

$$
\beta_{p(G), p(G)+m(G)+1}^{S}\left(S / J_{G}\right) \neq 0
$$

and together with Eq. (11), we get that $\beta_{p(G), p(G)+m(G)+1}^{S}\left(S / J_{G}\right)$ is an extremal Betti number. 
If $G$ is a complete graph, then $p(G)=n-1$ and $m(G)=0$. It follows from [10, Corollary $4.3]$ that $\beta_{n-1, n}^{S}\left(S / J_{G}\right)=n-1=f(G)-1$. We now assume that for every internal vertex $v, \operatorname{cdeg}_{G}(v)>2$. Therefore, $q \geq 2$, and as before we conclude that

$$
\operatorname{Tor}_{p\left(G_{A}[\bar{A}]\right), p\left(G_{A}[\bar{A}]\right)+m\left(G_{A}[\bar{A}]\right)+1}^{S_{A}}\left(\frac{S_{A}}{J_{G_{A}[\bar{A}]}}, K\right) \cong \operatorname{Tor}_{p(G), p(G)+m(G)+1}^{S}\left(\frac{S}{J_{G}}, K\right)
$$

Now, by induction, $\beta_{p\left(G_{A}[\bar{A}]\right), p\left(G_{A}[\bar{A}]\right)+m\left(G_{A}[\bar{A}]\right)+1}^{S_{A}}\left(S_{A} / J_{G_{A}[\bar{A}]}\right)=f\left(G_{A}[\bar{A}]\right)-1$. Since $f(G)=$ $f\left(G_{A}[\bar{A}]\right)$, we conclude that $\beta_{p(G), p(G)+m(G)+1}^{S}\left(S / J_{G}\right)=f(G)-1$.

Corollary 3.8. Let $G$ be a generalized block graph for which $G=G_{1} \cup \cdots \cup G_{s}$ is the decomposition of $G$ into indecomposable graphs. Then, $\beta_{p(G), p(G)+m(G)+1}^{S}\left(S / J_{G}\right)$ is an extremal Betti number of $S / J_{G}$.

Proof. Note that $m(G)=m\left(G_{1}\right)+\cdots+m\left(G_{s}\right)+s-1$. Now, the assertion follows from Proposition 3.5 and Theorem 3.7 .

As of now, the only lower bound known for regularity of binomial edge ideals of generalized block graphs is $\ell(G)$, which is a general lower bound given by Matsuda and Murai. If $H$ is a longest induced path of a generalized block graph $G$, then $\ell(G)=\ell(H)=m(H)+1 \leq$ $m(G)+1$. Thus, as an immediate consequence of Theorem 3.7, we obtain an improved lower bound for the regularity of binomial edge ideals of generalized block graphs.

Corollary 3.9. Let $G$ be a generalized block graph on $[n]$ with $c_{G}$ connected components. Then, $\operatorname{reg}\left(S / J_{G}\right) \geq m(G)+c_{G}$.

Proof. Let $G_{1}, \ldots, G_{c_{G}}$ be connected components of $G$. For $1 \leq i \leq c_{G}$, set $S_{i}=K\left[x_{j}, y_{j}\right.$ : $j \in V\left(G_{i}\right)$ ]. Then, for $1 \leq i \leq c_{G}$, by Corollary $3.8, \operatorname{reg}\left(S_{i} / J_{G_{i}}\right) \geq m\left(G_{i}\right)+1$. Note that $m(G)=m\left(G_{1}\right)+\cdots+m\left(G_{c_{G}}\right)$ and $S / J_{G} \simeq S_{1} / J_{G_{1}} \otimes \cdots \otimes S_{c_{G}} / J_{G_{c_{G}}}$. Thus, the minimal graded free resolution of $S / J_{G}$ is the tensor product of the minimal free resolutions of $S_{1} / J_{G_{1}}, \ldots, S_{c_{G}} / J_{G_{c_{G}}}$. Hence, $\operatorname{reg}\left(S / J_{G}\right)=\sum_{i=1}^{c_{G}} \operatorname{reg}\left(S_{i} / J_{G_{i}}\right) \geq m(G)+c_{G}$.

We now give an example of a connected chordal graph $G$ that is not a generalized block graph for which $\operatorname{reg}\left(S / J_{G}\right)<m(G)+1$.

Example 3.10. Let $G$ be a graph as shown in Fig. 2. Then, it can be seen that $G$ is a chordal graph that is not a generalized block graph. The minimal cut sets of $G$ are $\{2,3\},\{2,5\},\{3,5\}$. Therefore, $m(G)=3$. Using Macaulay2 [7], it can be seen that $\operatorname{reg}\left(S / J_{G}\right)=3<m(G)+1=4$.

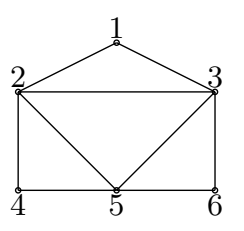

FiguRe 2 
Our aim is to classify generalized block graphs whose binomial edge ideals admit a unique extremal Betti number. Equivalently, we want to classify the generalized block graphs $G$ for which $\operatorname{reg}\left(S / J_{G}\right)=m(G)+1$. For that recall the definition of flower graph, introduced by Mascia and Rinaldo in [18]: a flower graph $F_{h, k}(v)$ is a connected graph obtained by gluing each of $h$ copies of the complete graph $K_{3}$ and $k$ copies of the star graph $K_{1,3}$ at a common vertex $v$, that is free in each of them. Now, we characterize generalized block graphs whose binomial edge ideals admit a unique extremal Betti number.

We denote by $\operatorname{iv}(G)$ the number of internal vertices of $G$.

Theorem 3.11. Let $G$ be a connected indecomposable generalized block graph. Then, the following are equivalent:

(1) $S / J_{G}$ admits a unique extremal Betti number.

(2) For any $v \in V(G), F_{h, k}(v)$ is not an induced subgraph of $G$ for every $h, k \geq 0$ with $h+k \geq 3$.

In this case, $\operatorname{reg}\left(S / J_{G}\right)=m(G)+1$.

Proof. (1) $\Longrightarrow(2):$ Suppose that for some $v \in V(G)$ and $h, k \geq 0$ with $h+k \geq 3$, $F_{h, k}(v)$ is an induced subgraph of $G$. It is enough to prove $\operatorname{reg}\left(S / J_{G}\right)>m(G)+1$. Let $H$ be an induced subgraph of $G$ obtained in the following way: for every minimal cut set $A$ with $|A| \geq 2$, remove $|A|-1$ elements of $A$ from $G$. Note that $H$ is a block graph with $\operatorname{iv}(H)=m(G)$ by [22, Proposition 2.1] and $F_{h, k}(v)$ is an induced subgraph of $H$. It follows from [11, Theorem 8] that $\operatorname{reg}\left(S / J_{H}\right)>$ iv $(H)+1$. Now, by virtue of [19, Corollary 2.2], $\operatorname{reg}\left(S / J_{G}\right) \geq \operatorname{reg}\left(S / J_{H}\right)>m(G)+1$.

$(2) \Longrightarrow(1):$ By Corollary 3.9 , it is enough to prove $\operatorname{reg}\left(S / J_{G}\right) \leq m(G)+1$. We prove this by induction on $m(G)$. If $m(G)=0$, then $G$ is a complete graph and the assertion is obvious. Assume that $m(G)>0$. Let $F_{1}, \ldots, F_{r}$ be a leaf order of $\mathcal{F}(\Delta(G))$. Let $A$ be the minimal cut set defined in the proof of Theorem 3.7. Then $G_{A}, G_{A}[\bar{A}]$ and $G[\bar{A}]$ are generalized block graphs. Note that $G_{A}$ and $G_{A}[\bar{A}]$ are generalized block graphs satisfying the hypothesis with $m\left(G_{A}\right)=m\left(G_{A}[\bar{A}]\right)=m(G)-1$. By induction, we have $\operatorname{reg}\left(S / J_{G_{A}}\right)=\operatorname{reg}\left(S / J_{G_{A}[\bar{A}]}\right) \leq m(G)$. As in the proof of Theorem [3.7, $G[\bar{A}]$ has $q+1$ connected components, say $H_{1}, \ldots, H_{q+1}$. Since $G$ has no induced $F_{h, k}$ with $h+k \geq 3$, at least $q-1$ components are isolated vertices. The two remaining components are a clique and a generalized block graph, say $H_{1}$, satisfying the assumption with $m\left(H_{1}\right) \leq m(G)-1$. Applying induction we obtain that $\operatorname{reg}\left(S / J_{G[\bar{A}]}\right) \leq m\left(H_{1}\right)+2 \leq m(G)+1$. Now, the assertion follows from the exact sequence (1) and [21, Corollary 18.7].

As an immediate consequence of Proposition 3.5 and Theorem 3.11, we have the following results:

Corollary 3.12. Let $G$ be a connected generalized block graph for which $G=G_{1} \cup \cdots \cup$ $G_{r}$ is the decomposition of $G$ into indecomposable graphs. Then, $S / J_{G}$ admits a unique extremal Betti number if and only if for each $i, S_{i} / J_{G_{i}}$ admits a unique extremal Betti number. Moreover, in this case $\operatorname{reg}\left(S / J_{G}\right)=m(G)+1$.

Recall that a caterpillar is a tree in which the removal of all pendant vertices leaves a path graph.

Corollary 3.13. Let $T$ be an indecomposable tree on $[n]$. Then, $S / J_{T}$ admits a unique extremal Betti number if and only if $T$ is a caterpillar. 
The following example illustrates that the lower bound $m(G)+1$ is not always attained.

Example 3.14. Let $G=F_{h, k}(v)$ be a flower graph with $h+k \geq 3$. Then, it follows from [18, Corollary 3.5] that $\operatorname{reg}\left(S / J_{G}\right)=m(G)+h+k-1>m(G)+1$.

\section{Regularity UPPER BOUND FOR GENERALIZED BLOCK GRAPH}

In this section, we give an improved upper bound for the regularity of binomial edge ideals of generalized block graphs. Let $u, v \in V(G)$ be such that $e=\{u, v\} \notin E(G)$, then we denote by $G_{e}$, the graph on the vertex set $V(G)$ and edge set $E\left(G_{e}\right)=E(G) \cup\{\{x, y\}: x, y \in$ $N_{G}(u)$ or $\left.x, y \in N_{G}(v)\right\}$. An edge $e$ is said to be a cut edge of $G$ if the number of connected components of $G \backslash e$ is larger than that of $G$.

We now recall a result from [15] that will be used repeatedly in this section.

Lemma 4.1. [15, Proposition 2.1] Let $G$ be a graph and e be a cut edge of $G$. Then,

$$
\operatorname{reg}\left(S / J_{G}\right) \leq \max \left\{\operatorname{reg}\left(S / J_{G \backslash e}\right), \operatorname{reg}\left(S / J_{(G \backslash e)_{e}}\right)+1\right\} .
$$

The degree of a vertex $v$ of $G$ is $\operatorname{deg}_{G}(v)=\left|N_{G}(v)\right|$. A vertex $v$ is said to be a pendant vertex if $\operatorname{deg}_{G}(v)=1$. For $v \in V(G)$, let $\operatorname{cdeg}_{G}(v)$ denote the number of maximal cliques of $G$ which contains $v$, and $\operatorname{pdeg}_{G}(v)$ denote the number of pendant vertices adjacent to $v$. Note that for every $v \in V(G), \operatorname{pdeg}_{G}(v) \leq \operatorname{cdeg}_{G}(v)$.

Remark 4.2. Let $G$ be a connected indecomposable generalized block graph which is not a star graph. If $e=\{u, v\}$ is an edge with pendant vertex $u$, then $(G \backslash e)_{e}=(G \backslash u)_{v} \sqcup\{u\}$, $\operatorname{cl}(G \backslash u)=\operatorname{cl}(G)-1, \operatorname{cl}\left((G \backslash u)_{v}\right)=\operatorname{cl}(G)-\operatorname{cdeg}_{G}(v)+1, J_{G \backslash e}=J_{G \backslash u}$ and $J_{(G \backslash e)_{e}}=J_{(G \backslash u)_{v}}$. Also, $(G \backslash e)_{e}$ and $G \backslash e$ are generalized block graphs other than star graphs.

A vertex $v \in V(G)$ with $\operatorname{pdeg}_{G}(v) \geq 1$ is said to be of type 1 if $\operatorname{cdeg}_{G}(v)=\operatorname{pdeg}_{G}(v)+1$, and of type 2 if $\operatorname{cdeg}_{G}(v) \geq \operatorname{pdeg}_{G}(v)+2$. We denote by $\alpha(G)$, the number of vertices of type 1 in $G$ and by $\operatorname{pv}(G)$, the number of pendant vertices of $G$.

Lemma 4.3. If $G$ is a connected indecomposable graph on $[n]$ with $\operatorname{pv}(G)>0$, then $\operatorname{pv}(G)-$ $\alpha(G)>0$.

Proof. First, we assume that $\alpha(G)=0$, then the claim follows. Now, assume that $\alpha(G)=$ $r>0$. Let $v_{1}, \ldots, v_{r}$ be all type 1 vertices of $G$. Since $G$ is an indecomposable graph, $\operatorname{cdeg}_{G}\left(v_{i}\right)=\operatorname{pdeg}_{G}\left(v_{i}\right)+1 \geq 3$, for $i \in[r]$. Thus, $\operatorname{pv}(G) \geq 2 \alpha(G)$ which completes the proof.

Proposition 4.4. Let $G=G_{1} \cup G_{2}$ be a decomposable graph such that $G_{1}$ and $G_{2}$ are indecomposable and let $S_{i}=K\left[x_{j}, y_{j}: j \in V\left(G_{i}\right)\right]$ for $i=1,2$. Suppose that one of the following conditions is satisfied:

(a) $G_{1}$ and $G_{2}$ are star graphs, or

(b) $G_{1}$ is a star graph, $G_{2}$ is not a star graph and $\operatorname{reg}\left(S_{2} / J_{G_{2}}\right) \leq \operatorname{cl}\left(G_{2}\right)+\alpha\left(G_{2}\right)-\operatorname{pv}\left(G_{2}\right)$, or

(c) for $i=1,2, G_{i}$ is not a star graph and $\operatorname{reg}\left(S_{i} / J_{G_{i}}\right) \leq \operatorname{cl}\left(G_{i}\right)+\alpha\left(G_{i}\right)-\operatorname{pv}\left(G_{i}\right)$.

Then $\operatorname{reg}\left(S / J_{G}\right) \leq \operatorname{cl}(G)+\alpha(G)-\operatorname{pv}(G)$.

Proof. (a) Since $G_{1}$ and $G_{2}$ are indecomposable star graphs, $\alpha(G)=2, \operatorname{pv}(G)=\operatorname{cl}(G)-2$. By Proposition 3.5 and [27, Theorem 4.1(a)], $\operatorname{reg}\left(S / J_{G}\right)=4=\operatorname{cl}(G)+\alpha(G)-\operatorname{pv}(G)$. Hence, the claim follows. 
(b) Let $V\left(G_{1}\right) \cap V\left(G_{2}\right)=\{u\}$. First, assume that $u$ is not a pendant vertex of $G_{2}$. Therefore, $\alpha(G)=\alpha\left(G_{2}\right)+1$ and $\operatorname{pv}(G)=\operatorname{pv}\left(G_{1}\right)+\operatorname{pv}\left(G_{2}\right)-1$. Note that $\operatorname{cl}(G)=$ $\operatorname{cl}\left(G_{1}\right)+\operatorname{cl}\left(G_{2}\right)$ and $\operatorname{cl}\left(G_{1}\right)=\operatorname{pv}\left(G_{1}\right)$. By [27, Theorem 4.1(a)], $\operatorname{reg}\left(S_{1} / J_{G_{1}}\right)=2$. Hence, Proposition 3.5 yields that $\operatorname{reg}\left(S / J_{G}\right) \leq \operatorname{cl}(G)+\alpha(G)-\operatorname{pv}(G)$. We now assume that $u$ is a pendant vertex of $G_{2}$. Note that $\operatorname{pv}(G)=\operatorname{pv}\left(G_{1}\right)+\operatorname{pv}\left(G_{2}\right)-2$ and $\operatorname{cl}\left(G_{1}\right)=\operatorname{pv}\left(G_{1}\right)$. Let $v \in N_{G_{2}}(u)$. If $v$ is of type 1 in $G_{2}$, then $\alpha(G)=\alpha\left(G_{2}\right)$, and hence, the claim follows from Proposition 3.5. If $v$ is of type 2 in $G_{2}$, then $\alpha(G)=\alpha\left(G_{2}\right)+1$, and hence, by Proposition 3.5, $\operatorname{reg}\left(S / J_{G}\right) \leq \operatorname{cl}(G)+\alpha(G)-\operatorname{pv}(G)$.

(c) Let $V\left(G_{1}\right) \cap V\left(G_{2}\right)=\{u\}$. Observe that, $\operatorname{cl}(G)=\operatorname{cl}\left(G_{1}\right)+\operatorname{cl}\left(G_{2}\right)$. If $\operatorname{deg}_{G_{1}}(u), \operatorname{deg}_{G_{2}}(u)>$ 1, then $\alpha(G)=\alpha\left(G_{1}\right)+\alpha\left(G_{2}\right)$ and $\operatorname{pv}(G)=\operatorname{pv}\left(G_{1}\right)+\operatorname{pv}\left(G_{2}\right)$. Thus, by Proposition 3.5, $\operatorname{reg}\left(S / J_{G}\right)=\operatorname{reg}\left(S_{1} / J_{G_{1}}\right)+\operatorname{reg}\left(S_{2} / J_{G_{2}}\right) \leq \operatorname{cl}(G)+\alpha(G)-\operatorname{pv}(G)$.

Assume that $u$ is a pendant vertex of $G_{1}$, let $N_{G_{1}}(u)=\left\{u_{1}\right\}$ and $\operatorname{deg}_{G_{2}}(u)>1$ (or vice versa). Then $\operatorname{pv}(G)=\operatorname{pv}\left(G_{1}\right)+\operatorname{pv}\left(G_{2}\right)-1$. If $u_{1}$ is of type 1 in $G_{1}$, then we have $\alpha(G)=$ $\alpha\left(G_{1}\right)+\alpha\left(G_{2}\right)-1$. If $u_{1}$ is of type 2 in $G_{1}$, then we have $\alpha(G)=\alpha\left(G_{1}\right)+\alpha\left(G_{2}\right)$. Hence, by Proposition 3.5, $\operatorname{reg}\left(S / J_{G}\right)=\operatorname{reg}\left(S / J_{G_{1}}\right)+\operatorname{reg}\left(S / J_{G_{2}}\right) \leq \operatorname{cl}(G)+\alpha(G)-\operatorname{pv}(G)$.

Now, assume that for $i=1,2, u$ is a pendant vertex of $G_{i}$, let $N_{G_{i}}(u)=\left\{u_{i}\right\}$. Then, $\operatorname{pv}(G)=\operatorname{pv}\left(G_{1}\right)+\operatorname{pv}\left(G_{2}\right)-2$. If both $u_{1}$ and $u_{2}$ are of type 1 in $G_{1}$ and $G_{2}$, respectively, then we have $\alpha(G)=\alpha\left(G_{1}\right)+\alpha\left(G_{2}\right)-2$. If both $u_{1}$ and $u_{2}$ are of type 2 in $G_{1}$ and $G_{2}$, respectively, then $\alpha(G)=\alpha\left(G_{1}\right)+\alpha\left(G_{2}\right)$. If $u_{1}$ is of type 1 in $G_{1}$ and $u_{2}$ is of type 2 in $G_{2}$ (or vice versa), then we have $\alpha(G)=\alpha\left(G_{1}\right)+\alpha\left(G_{2}\right)-1$. Hence, Proposition 3.5 yields $\operatorname{reg}\left(S / J_{G}\right)=\operatorname{reg}\left(S_{1} / J_{G_{1}}\right)+\operatorname{reg}\left(S_{2} / J_{G_{2}}\right) \leq \operatorname{cl}(G)+\alpha(G)-\operatorname{pv}(G)$.

We now obtain a tight upper bound for the regularity of binomial edge ideals of connected indecomposable generalized block graphs.

Theorem 4.5. Let $G$ be a connected indecomposable generalized block graph on $[n]$ which is not a star graph. Then, $\operatorname{reg}\left(S / J_{G}\right) \leq \operatorname{cl}(G)+\alpha(G)-\operatorname{pv}(G)$.

Proof. Let $k(G)=\left|\left\{v: \operatorname{pdeg}_{G}(v) \geq 1\right\}\right|$. We proceed by induction on $k(G)+m(G) \geq 0$. For $k(G)=0, \operatorname{pv}(G)=\alpha(G)=0$, and hence, the result is immediate from [24, Theorem 3.5] or [16, Theorem 3.15]. If $m(G)=0$, then $G$ is a complete graph and the assertion is obvious. Assume that $k=k(G)>0, m(G)>0$ and the assertion is true up to $k(G)+m(G)-1$.

Let $v_{1}, \ldots, v_{k} \in V(G)$ be such that for each $i=1, \ldots, k, \operatorname{pdeg}\left(v_{i}\right)=r_{i} \geq 1$. For each $i=1, \ldots, k$, let $e_{i, 1}=\left\{v_{i}, w_{i, 1}\right\}, \ldots, e_{i, r_{i}}=\left\{v_{i}, w_{i, r_{i}}\right\}$ be pendant edges incident to $v_{i}$. Since $G$ is an indecomposable graph, $\operatorname{cdeg}_{G}\left(v_{i}\right)=s_{i} \geq 3$.

We proceed by induction on $r_{k}$. If $r_{k}=1$, then $v_{k}$ is of type 2 . Notice that $k\left(\left(G \backslash w_{k, 1}\right)_{v_{k}}\right)=$ $k(G)-1$ and $m\left(\left(G \backslash w_{k, 1}\right)_{v_{k}}\right)=m(G)-1$. Thus, by induction on $k(G)+m(G)$ and Remark 4.2, we have

$$
\begin{aligned}
\operatorname{reg}\left(S / J_{\left.\left(G \backslash e_{k, 1}\right)_{e_{k, 1}}\right)}\right. & =\operatorname{reg}\left(S / J_{\left(G \backslash w_{k, 1}\right)_{v_{k}}}\right) \\
& \leq \operatorname{cl}\left(\left(G \backslash w_{k, 1}\right)_{v_{k}}\right)+\alpha\left(\left(G \backslash w_{k, 1}\right)_{v_{k}}\right)-\operatorname{pv}\left(\left(G \backslash w_{k, 1}\right)_{v_{k}}\right) \\
& =\operatorname{cl}(G)-\operatorname{cdeg}_{G}\left(v_{k}\right)+\alpha(G)-\operatorname{pv}(G)+2 \\
& \leq \operatorname{cl}(G)+\alpha(G)-\operatorname{pv}(G)-1
\end{aligned}
$$

If cdeg ${ }_{G}\left(v_{k}\right)=3$, then $G \backslash w_{k, 1}=G_{1} \cup G_{2}$ is a decomposable graph. If $G_{i}$ is not a star graph, then $k\left(G_{i}\right) \leq k(G)$ and $m\left(G_{i}\right)<m(G)$, and hence, by induction on $k(G)+m(G)$, we have $\operatorname{reg}\left(S_{i} / J_{G_{i}}\right) \leq \operatorname{cl}\left(G_{i}\right)+\alpha\left(G_{i}\right)-\operatorname{pv}\left(G_{i}\right)$. Note that $G$ satisfies the assumption of Proposition 
4.4. It follows from Proposition 4.4 that

$$
\begin{aligned}
\operatorname{reg}\left(S / J_{G \backslash e_{k, 1}}\right) & =\operatorname{reg}\left(S / J_{G \backslash w_{k, 1}}\right) \\
& \leq \operatorname{cl}\left(G \backslash w_{k, 1}\right)+\alpha\left(G \backslash w_{k, 1}\right)-\operatorname{pv}\left(G \backslash w_{k, 1}\right) \\
& =\operatorname{cl}(G)+\alpha(G)-\operatorname{pv}(G) .
\end{aligned}
$$

If $\operatorname{cdeg}_{G}\left(v_{k}\right)>3$, then $G \backslash w_{k, 1}$ is an indecomposable generalized block graph with $k(G \backslash$ $\left.w_{k, 1}\right)=k(G)-1$ and $m\left(G \backslash w_{k, 1}\right)=m(G)$. Thus, by induction on $k(G)+m(G)$ and Remark 4.2, we have

$$
\begin{aligned}
\operatorname{reg}\left(S / J_{G \backslash e_{k, 1}}\right) & =\operatorname{reg}\left(S / J_{G \backslash w_{k, 1}}\right) \\
& \leq \operatorname{cl}\left(G \backslash w_{k, 1}\right)+\alpha\left(G \backslash w_{k, 1}\right)-\operatorname{pv}\left(G \backslash w_{k, 1}\right) \\
& =\operatorname{cl}(G)+\alpha(G)-\operatorname{pv}(G) .
\end{aligned}
$$

In both the cases, we get $\operatorname{reg}\left(S / J_{G \backslash e_{k, 1}}\right) \leq \operatorname{cl}(G)+\alpha(G)-\operatorname{pv}(G)$. Hence, by Lemma 4.1, we have

$$
\operatorname{reg}\left(S / J_{G}\right) \leq \operatorname{cl}(G)+\alpha(G)-\operatorname{pv}(G) .
$$

Assume now that $r_{k}>1$. By Remark 4.2, we get $k\left(\left(G \backslash w_{k, r_{k}}\right)_{v_{k}}\right)=k(G)-1, \operatorname{pv}((G \backslash$ $\left.\left.w_{k, r_{k}}\right)_{v_{k}}\right)=\operatorname{pv}(G)-r_{k}, \operatorname{cl}\left(\left(G \backslash w_{k, r_{k}}\right)_{v_{k}}\right)=\operatorname{cl}(G)-\operatorname{cdeg}_{G}\left(v_{k}\right)+1$ and $m\left(\left(G \backslash w_{k, r_{k}}\right)_{v_{k}}\right)<m(G)$.

Case (1): $v_{k}$ is of type 2 in $G$.

Thus, $\alpha\left(\left(G \backslash w_{k, r_{k}}\right)_{v_{k}}\right)=\alpha(G), \operatorname{cdeg}_{G}\left(v_{k}\right)-r_{k} \geq 2$, and hence, by Remark 4.2 and induction on $k(G)+m(G)$, we have

$$
\begin{aligned}
\operatorname{reg}\left(S / J_{\left.\left(G \backslash e_{k, r_{k}}\right)_{e_{k, r_{k}}}\right)}\right. & =\operatorname{reg}\left(S / J_{\left.\left(G \backslash w_{k, r_{k}}\right)_{v_{k}}\right)}\right. \\
& \leq \operatorname{cl}\left(\left(G \backslash w_{k, r_{k}}\right)_{v_{k}}\right)+\alpha\left(\left(G \backslash w_{k, r_{k}}\right)_{v_{k}}\right)-\operatorname{pv}\left(\left(G \backslash w_{k, r_{k}}\right)_{v_{k}}\right) \\
& =\operatorname{cl}(G)-\operatorname{cdeg}_{G}\left(v_{k}\right)+1+\alpha(G)-\operatorname{pv}(G)+r_{k} \\
& \leq \operatorname{cl}(G)+\alpha(G)-\operatorname{pv}(G)-1 .
\end{aligned}
$$

Note that $v_{k}$ is of type 2 in $G \backslash w_{k, r_{k}}$ and $G \backslash w_{k, r_{k}}$ is indecomposable. It follows from Remark 4.2, and induction on $r_{k}$ that

$$
\begin{aligned}
\operatorname{reg}\left(S / J_{G \backslash e_{k, r_{k}}}\right) & =\operatorname{reg}\left(S / J_{G \backslash w_{k, r_{k}}}\right) \\
& \leq \operatorname{cl}\left(G \backslash w_{k, r_{k}}\right)+\alpha\left(G \backslash w_{k, r_{k}}\right)-\operatorname{pv}\left(G \backslash w_{k, r_{k}}\right) \\
& =\operatorname{cl}(G)+\alpha(G)-\operatorname{pv}(G) .
\end{aligned}
$$

Thus, by Lemma 4.1, $\operatorname{reg}\left(S / J_{G}\right) \leq \operatorname{cl}(G)+\alpha(G)-\operatorname{pv}(G)$.

Case (2): $v_{k}$ is of type 1 in $G$.

Thus, $\alpha\left(\left(G \backslash w_{k, r_{k}}\right)_{v_{k}}\right)=\alpha(G)-1, \operatorname{cdeg}_{G}\left(v_{k}\right)-r_{k}=1$, and hence, by Remark 4.2 and induction on $k(G)+m(G)$,

$$
\begin{aligned}
\operatorname{reg}\left(S / J_{\left.\left(G \backslash e_{k, r_{k}}\right)_{e_{k, r_{k}}}\right)}\right. & =\operatorname{reg}\left(S / J_{\left.\left(G \backslash w_{k, r_{k}}\right)_{v_{k}}\right)}\right. \\
& \leq \operatorname{cl}\left(\left(G \backslash w_{k, r_{k}}\right)_{v_{k}}\right)+\alpha\left(\left(G \backslash w_{k, r_{k}}\right)_{v_{k}}\right)-\operatorname{pv}\left(\left(G \backslash w_{k, r_{k}}\right)_{v_{k}}\right) \\
& =\operatorname{cl}(G)-\operatorname{cdeg}_{G}\left(v_{k}\right)+\alpha(G)-\operatorname{pv}(G)+r_{k} \\
& =\operatorname{cl}(G)+\alpha(G)-\operatorname{pv}(G)-1 .
\end{aligned}
$$

Note that $v_{k}$ is of type 1 in $G \backslash w_{k, r_{k}}$. If $r_{k}=2$, then $G \backslash w_{k, r_{k}}$ is a decomposable graph with decomposition $\left(G \backslash\left\{w_{k, 1}, w_{k, 2}\right\}\right) \cup\left\{e_{k, 1}\right\}$. Set $H=G \backslash\left\{w_{k, 1}, w_{k, 2}\right\}$. If $H$ is not a star graph, then $k(H) \leq k(G)$ and $m(H)<m(G)$. Thus, by induction on $k(G)+m(G)$, 


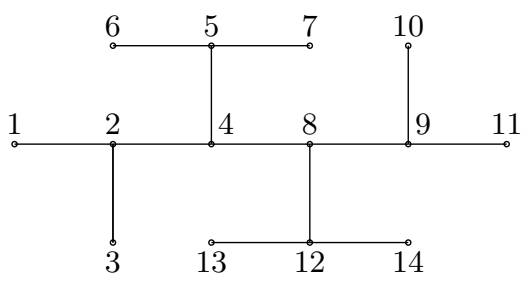

FiguRE 3

$\operatorname{reg}\left(S / J_{H}\right) \leq \operatorname{cl}(H)+\alpha(H)-\operatorname{pv}(H)$. Note that $\operatorname{cl}(H)=\operatorname{cl}(G)-2$. If $\operatorname{deg}_{H}\left(v_{k}\right)=1$, then $\operatorname{pv}(H)=p(G)-1$ and $\alpha(H) \leq \alpha(G)$, and if $\operatorname{deg}_{H}\left(v_{k}\right)>1$, then $\operatorname{pv}(H)=\operatorname{pv}(G)-2$ and $\alpha(H)=\alpha(G)-1$. Therefore, in both cases, $\operatorname{reg}\left(S / J_{H}\right) \leq \operatorname{cl}(G)+\alpha(G)-\operatorname{pv}(G)-1$. Now, it follows from Proposition 3.5 that $\operatorname{reg}\left(S / J_{G \backslash e_{k, r_{k}}}\right) \leq \operatorname{cl}(G)+\alpha(G)-\operatorname{pv}(G)$. If $G \backslash\left\{w_{k, 1}, w_{k, 2}\right\}$ is a star graph, then $\alpha(G)=2$ and $\operatorname{cl}(G)-\operatorname{pv}(G)=1$. By Proposition 3.5, $\operatorname{reg}\left(S / J_{G \backslash e_{k, r_{k}}}\right)=3$. Thus, $\operatorname{reg}\left(S / J_{G \backslash e_{k, r_{k}}}\right) \leq \operatorname{cl}(G)+\alpha(G)-\operatorname{pv}(G)$.

If $r_{k}>2$, then $G \backslash w_{k, r_{k}}$ is indecomposable. By induction on $r_{k}$, we have

$$
\begin{aligned}
\operatorname{reg}\left(S / J_{G \backslash e_{k, r_{k}}}\right) & =\operatorname{reg}\left(S / J_{G \backslash w_{k, r_{k}}}\right) \\
& \leq \operatorname{cl}\left(G \backslash w_{k, r_{k}}\right)+\alpha\left(G \backslash w_{k, r_{k}}\right)-\operatorname{pv}\left(G \backslash w_{k, r_{k}}\right) \\
& =\operatorname{cl}(G)+\alpha(G)-\operatorname{pv}(G)-1 .
\end{aligned}
$$

Thus, for $r_{k} \geq 2, \operatorname{reg}\left(S / J_{G \backslash e_{k, r_{k}}}\right) \leq \operatorname{cl}(G)+\alpha(G)-\operatorname{pv}(G)$. Consequently, by Lemma 4.1, $\operatorname{reg}\left(S / J_{G}\right) \leq \operatorname{cl}(G)+\alpha(G)-\operatorname{pv}(G)$. Hence, the assertion follows.

The following example illustrates that the upper bound is not always attained in Theorem 4.5.

Example 4.6. Let $G$ be a tree as shown in Fig. 3. Notice that $\operatorname{cl}(G)=13, \alpha(G)=4$ and $\operatorname{pv}(G)=8$. Thus, $\operatorname{cl}(G)+\alpha(G)-\operatorname{pv}(G)=9$. Using Macaulay2 [7], we get $\operatorname{reg}\left(S / J_{G}\right)=8$.

As an immediate consequence, we have the following:

Corollary 4.7. Let $T$ be an indecomposable tree on $[n]$ which is not a star graph. Then, $\operatorname{reg}\left(S / J_{T}\right) \leq \operatorname{cl}(T)+\alpha(T)-\operatorname{pv}(T)$.

Finally we show two classes of block graphs that attain the upper bound $\mathrm{cl}(G)+\alpha(G)-$ $\operatorname{pv}(G)$.

Corollary 4.8. If $G$ is an indecomposable caterpillar that is not a star graph or a flower graph, then $\operatorname{reg}\left(S / J_{G}\right)=\operatorname{cl}(G)+\alpha(G)-\operatorname{pv}(G)$.

Proof. First, let $G$ be an indecomposable caterpillar that is not a star graph. Then $m(G)+$ $1=\operatorname{cl}(G)+\alpha(G)-\operatorname{pv}(G)$. Therefore, by Corollaries 3.13 and 4.7, $\operatorname{reg}\left(S / J_{G}\right)=\operatorname{cl}(G)+$ $\alpha(G)-\operatorname{pv}(G)$.

Now, let $G=F_{h, k}(v)$ be a flower graph. Then $\operatorname{cl}(G)+\alpha(G)-\operatorname{pv}(G)=m(G)+\operatorname{cdeg}_{G}(v)-$ $1=h+2 k$. Thus, by virtue of [18, Corollary 3.5], $\operatorname{reg}\left(S / J_{G}\right)=\operatorname{cl}(G)+\alpha(G)-\operatorname{pv}(G)$.

Acknowledgements: The author is grateful to his advisor, Prof. A. V. Jayanthan for the constant support, valuable ideas, and suggestions. The author thanks the National Board for 
Higher Mathematics, India, for the financial support. The author also wishes to express his sincere gratitude to the anonymous referee whose comments help to improve the exposition in great detail.

\section{REFERENCES}

[1] Josep Àlvarez Montaner. Local cohomology of binomial edge ideals and their generic initial ideals. Collect. Math., 71(2):331-348, 2020.

[2] Arindam Banerjee and Luis Núñez Betancourt. Graph connectivity and binomial edge ideals. Proc. Amer. Math. Soc., 145(2):487-499, 2017.

[3] Davide Bolognini, Antonio Macchia, and Francesco Strazzanti. Binomial edge ideals of bipartite graphs. European J. Combin., 70:1-25, 2018.

[4] J. A. Eagon and D. G. Northcott. Ideals defined by matrices and a certain complex associated with them. Proc. Roy. Soc. Ser. A, 269:188-204, 1962.

[5] Viviana Ene, Jürgen Herzog, and Takayuki Hibi. Cohen-Macaulay binomial edge ideals. Nagoya Math. J., 204:57-68, 2011.

[6] Viviana Ene and Andrei Zarojanu. On the regularity of binomial edge ideals. Math. Nachr., 288(1):1924, 2015.

[7] Daniel R. Grayson and Michael E. Stillman. Macaulay2, a software system for research in algebraic geometry. Available at http://www.math.uiuc.edu/Macaulay2/

[8] Jürgen Herzog and Takayuki Hibi. Monomial ideals, volume 260 of Graduate Texts in Mathematics. Springer-Verlag London, Ltd., London, 2011.

[9] Jürgen Herzog, Takayuki Hibi, Freyja Hreinsdóttir, Thomas Kahle, and Johannes Rauh. Binomial edge ideals and conditional independence statements. Adv. Appl. Math., 45(3):317-333, 2010.

[10] Jürgen Herzog, Dariush Kiani, and Sara Saeedi Madani. The linear strand of determinantal facet ideals. Michigan Math. J., 66(1):107-123, 2017.

[11] Jürgen Herzog and Giancarlo Rinaldo. On the extremal Betti numbers of binomial edge ideals of block graphs. Electron. J. Combin., 25(1):Paper 1.63, 10, 2018.

[12] A. V. Jayanthan and Arvind Kumar. Regularity of binomial edge ideals of Cohen-Macaulay bipartite graphs. Comm. Algebra, 47(11):4797-4805, 2019.

[13] A. V. Jayanthan, N. Narayanan, and B. V. Raghavendra Rao. Regularity of binomial edge ideals of certain block graphs. Proc. Indian Acad. Sci. Math. Sci., 129(3):Art. 36, 10, 2019.

[14] Dariush Kiani and Sara Saeedi Madani. Some Cohen-Macaulay and unmixed binomial edge ideals. Comm. Algebra, 43(12):5434-5453, 2015.

[15] Dariush Kiani and Sara Saeedi Madani. The Castelnuovo-Mumford regularity of binomial edge ideals. J. Combin. Theory Ser. A, 139:80-86, 2016.

[16] Arvind Kumar. Binomial edge ideals and bounds for their regularity. to appear in J. Algebr. Comb., 2020.

[17] Arvind Kumar and Rajib Sarkar. Hilbert series of binomial edge ideals. Comm. Algebra, 47(9):3830$3841,2019$.

[18] Carla Mascia and Giancarlo Rinaldo. Krull dimension and regularity of binomial edge ideals of block graphs. to appear in J. Alg. Appl., https://doi.org/10. 1142/S0219498820501339, 2020.

[19] Kazunori Matsuda and Satoshi Murai. Regularity bounds for binomial edge ideals. J. Commut. Algebra, $5(1): 141-149,2013$.

[20] Masahiro Ohtani. Graphs and ideals generated by some 2-minors. Comm. Algebra, 39(3):905-917, 2011.

[21] Irena Peeva. Graded syzygies, volume 14 of Algebra and Applications. Springer-Verlag London, Ltd., London, 2011.

[22] Asia Rauf and Giancarlo Rinaldo. Construction of Cohen-Macaulay binomial edge ideals. Comm. Algebra, 42(1):238-252, 2014.

[23] Giancarlo Rinaldo. Cohen-Macaulay binomial edge ideals of cactus graphs. J. Algebra Appl., 18(4):1950072, 18, 2019.

[24] M. Rouzbahani Malayeri, S. Saeedi Madani, and D. Kiani. Regularity of binomial edge ideals of chordal graphs. preprint, http: //adsabs. harvard. edu/abs/2018arXiv181003119R, 2018. 
[25] Sara Saeedi Madani and Dariush Kiani. Binomial edge ideals of graphs. Electron. J. Combin., 19(2):Paper 44, 6, 2012.

[26] Sara Saeedi Madani and Dariush Kiani. On the binomial edge ideal of a pair of graphs. Electron. J. Combin., 20(1):Paper 48, 13, 2013.

[27] Peter Schenzel and Sohail Zafar. Algebraic properties of the binomial edge ideal of a complete bipartite graph. An. Ştiinţ. Univ. "Ovidius" Constanţa Ser. Mat., 22(2):217-237, 2014.

E-mail address: arvkumar11@gmail.com

Department of Mathematics, Indian Institute of Technology Madras, Chennai, INDiA 600036 